\title{
PARAMETER ESTIMATES FOR LINEAR PARTIAL DIFFERENTIAL EQUATIONS WITH FRACTIONAL BOUNDARY NOISE*
}

\author{
BOHDAN MASLOWSKI ${ }^{\dagger}$ AND JAN POSPÍŠIL
}

\begin{abstract}
Parameter-dependent linear evolution equations with a fractional noise in the boundary conditions are studied. Ergodic-type theorems for stationary and non-stationary solutions are verified and used to prove the strong consistency of a suitably defined family of estimators.
\end{abstract}

Key words: parameter identification, ergodicity, stochastic partial differential equations, fractional Brownian motion, fractional Ornstein-Uhlenbeck process

1. Introduction. Evolution equations perturbed by a fractional Gaussian noise have been studied in recent years by several authors, e.g. Duncan, Maslowski and Pasik-Duncan [4], [5] and [6]; Grecksch and Anh [7] Maslowski and Nualart [11], and others. In these papers (with the exception of a section in [4]) equations perturbed by distributed noise are considered. In the present paper we deal with a parabolic problem (typically, the heat equation) perturbed by a boundary noise, that is, the boundary conditions (for example, of the Dirichlet or Neumann type) involve the fractional noise, the generalized derivative of a fractional Brownian motion. More specifically, consider the problem

$$
\frac{\partial y}{\partial t}(t, \xi)=\alpha \Delta y(t, \xi), \quad(t, \xi) \in \mathbf{R}_{+} \times \mathcal{O},
$$

where $\mathcal{O}$ is a bounded domain in the Euclidean space with a smooth boundary and $\alpha>0$ is an unknown parameter. We consider the initial condition $y(0, \xi)=x_{0}(\xi)$ and boundary conditions, for example,

$$
y(t, \xi)=\eta^{H}(t, \xi), \quad(t, \xi) \in \mathbf{R}_{+} \times \partial \mathcal{O},
$$

where $\eta^{H}$ denotes formally the derivative of a space-dependent process that is fractional Brownian motion in time, with the Hurst parameter $H \in(0,1)$.

Boundary (and pointwise) noise problems have been considered (for a fixed value of the parameter) in numerous papers in case when $H=1 / 2$, i.e. the noise is white in time (cf. [2], Section 13 and the references therein). For the "fractional" case when

\footnotetext{
* This work was partially supported by the GACR Grant 201/07/0237 and by the MSMT Research Plan MSM 4977751301.

$\dagger$ Institute of Mathematics, Czech Academy of Sciences, Žitná 25, 11567 Prague 1, Czech Republic, E-mail: maslow@math.cas.cz

${ }^{\ddagger}$ University of West Bohemia, Faculty of Applied Sciences, Department of Mathematics, Univerzitní 22, 30614 Plzeň, Czech Republic, E-mail: jan.pospisil@kma.zcu.cz
} 
$H>1 / 2$, the basic theory may be found in [4], Section 5 , where results on existence, uniqueness and pathwise regularity of solutions are proven.

The problem of estimating parameters in stochastic equations with fractional noises has been studied also only recently. Estimation for some finite-dimensional stochastic equations based on discrete observations was presented by Prakasa Rao in [18]. He also investigated the asymptotic properties of the maximum likelihood estimator and the Bayes estimator of the drift parameter in stochastic equations driven by fractional Brownian motion, see [19] and [20]. In infinite-dimensional case (for stochastic PDEs) the problem has been studied in [12] (for linear equations). For stochastic parabolic equation with a scalar multiplicative fractional Brownian motion was also mentioned in [5]. Of course, the problem has been rather extensively studied in the important particular case of white noise $(H=1 / 2)$. There exist several methods mainly of statistical origin. Let us mention at least the so called maximum likelihood estimates method. Huebner and Rozovskii used it in [9] to estimate parameters from a continuous observation of a solution to stochastic parabolic equations driven by Wiener process. Khasminskii and Milstein studied in [10] the estimation of the linearised drift for nonlinear SDEs.

This paper is in principle a continuation of an earlier authors' paper [12] where similar problems have been studied for equations perturbed by distributed fractional noise. In order to treat the problem rigorously, at first a mathematically correct model is introduced (following [4]) and some results on existence and ergodicity of strictly stationary solutions are given for the equation with a fixed parameter value. The abstract model that is considered is rather general and as particular cases it may cover a wide range of linear stochastic PDEs (of parabolic type) as well as the finitedimensional equations. For the parameter dependent equation at first the dependence of the equilibrium measure on the parameter must be specified. This is important for our definition of estimators. In this respect the results are different from equations with distributed noise ([12]) because in the abstract model (or the infinite-dimensional formulation of the boundary noise problem) the parameter $\alpha$ automatically appears also in the noise term.

Section 2 of the paper contains some preliminaries, basic definitions and notation are given and the infinite dimensional formulation of the problem is presented. Section 3 is devoted to ergodic results: Existence and ergodicity of a stationary solution is stated (the proofs of these results are completely analogous to the case considered in [12] and are only sketched) and a theorem on ergodic behaviour of solutions with an arbitrary initial datum is proved (this result is however different from those considered in [12] due to the different form of the equation). In Section 4, a class of parameter estimator is proposed and the strong consistency is proved. Finally, in the last section 
the specific problems are considered: The heat equation as above with boundary noise of either Dirichlet and Neumann type. Conditions of the general theory from the preceding sections are verified in these cases.

2. Preliminaries. Let $K_{H}(t, s)$ for $0 \leq s \leq t \leq T$ be the kernel function

$$
K_{H}(t, s)=c_{H}(t-s)^{H-\frac{1}{2}}+c_{H}\left(\frac{1}{2}-H\right) \int_{s}^{t}(u-s)^{H-\frac{3}{2}}\left(1-\left(\frac{s}{u}\right)^{\frac{1}{2}-H}\right) d u
$$

where

$$
c_{H}=\left[\frac{2 H \Gamma\left(H+\frac{1}{2}\right) \Gamma\left(\frac{3}{2}-H\right)}{\Gamma(2-2 H)}\right]^{\frac{1}{2}},
$$

where $\Gamma(\cdot)$ is the gamma function and $H \in(0,1)$.

Following [1] and [3], we will define a stochastic integral of a deterministic $V$ valued function with respect to a scalar fractional Brownian motion $\left(\beta^{H}(t), t \in \mathbf{R}\right)$.

Let $\mathcal{K}_{H}^{*}: \mathcal{E} \rightarrow L^{2}([0, T], V)$ be the linear operator given by

$$
\mathcal{K}_{H}^{*} \varphi(t):=\varphi(t) K_{H}(T, t)+\int_{t}^{T}(\varphi(s)-\varphi(t)) \frac{\partial K_{H}}{\partial s}(s, t) d s
$$

for $\varphi \in \mathcal{E}$ where $\mathcal{E}$ is the linear space of $V$-valued step functions on [0,T], that is,

$$
\varphi(t)=\sum_{i=1}^{n-1} x_{i} \mathbb{1}_{\left[t_{i}, t_{i+1}\right)}(t)
$$

where $x_{i} \in V, i \in\{1, \ldots, n-1\}$ and $0=t_{1}<\cdots<t_{n}=T$.

Setting

$$
\int_{0}^{T} \varphi d \beta^{H}:=\sum_{i=1}^{n} x_{i}\left(\beta^{H}\left(t_{i+1}\right)-\beta^{H}\left(t_{i}\right)\right)
$$

it follows directly that

$$
\mathbb{E}\left|\int_{0}^{T} \varphi d \beta^{H}\right|_{V}^{2}=\left|\mathcal{K}_{H}^{*} \varphi\right|_{L^{2}([0, T], V)}^{2} .
$$

Let $\left(\mathcal{H},|\cdot|_{\mathcal{H}},\langle\cdot, \cdot,\rangle_{\mathcal{H}}\right)$ be the Hilbert space obtained by the completion of the preHilbert space $\mathcal{E}$ with respect to the inner product

$$
\langle\varphi, \psi\rangle_{\mathcal{H}}:=\left\langle\mathcal{K}_{H}^{*} \varphi, \mathcal{K}_{H}^{*} \psi\right\rangle_{L^{2}([0, T], V)}
$$

for $\varphi, \psi \in \mathcal{E}$. The stochastic integral (1) is extended to $\mathcal{H}$ by the isometry (2). Thus $\mathcal{H}$ is the space of integrable functions and it is useful to obtain some more specific information. 
If $H \in\left(\frac{1}{2}, 1\right)$, then it is easily verified that $\mathcal{H} \supset \tilde{\mathcal{H}}$ where $\tilde{\mathcal{H}}$ is the Banach space of Borel measurable functions with the norm $|\cdot|_{\tilde{\mathcal{H}}}$ given by

$$
|\varphi|_{\tilde{\mathcal{H}}}^{2}:=\int_{0}^{T} \int_{0}^{T}|\varphi(u)|_{V}|\varphi(v)|_{V} \phi(u-v) d u d v
$$

where $\phi(u)=H(2 H-1)|u|^{2 H-2}$. It may be verified that $\tilde{\mathcal{H}} \supset L^{1 / H}([0, T], V)$ and consequently $\tilde{\mathcal{H}} \supset L^{2}([0, T], V)$. If $\varphi \in \tilde{\mathcal{H}}$ and $H>\frac{1}{2}$, then

$$
\mathbb{E}\left|\int_{0}^{T} \varphi d \beta^{H}\right|_{V}^{2}=\int_{0}^{T} \int_{0}^{T}\langle\varphi(u), \varphi(v)\rangle_{V} \phi(u-v) d u d v .
$$

If $H \in\left(0, \frac{1}{2}\right)$, the space of integrable functions is smaller than $L^{2}([0, T], V)$. It is known that $\mathcal{H} \supset H^{1}([0, T], V)$ (e.g. [8] Lemma 5.20) and $\mathcal{H} \supset C^{\beta}([0, T], V)$ for each $\beta>\frac{1}{2}-H$. If $H \in\left(0, \frac{1}{2}\right)$, then the linear operator $\mathcal{K}_{H}^{*}$ can be described by the composition

$$
\mathcal{K}_{H}^{*} \varphi(t)=c_{H} t^{\frac{1}{2}-H} D_{T-}^{\frac{1}{2}-H}\left(u_{H-\frac{1}{2}} \varphi\right)
$$

where

$$
\left(D_{T-}^{\alpha} \psi\right)(t)=\frac{1}{\Gamma(1-\alpha)}\left(\frac{\psi(t)}{(T-t)^{\alpha}}+\alpha \int_{t}^{T} \frac{\psi(s)-\psi(t)}{(s-t)^{\alpha+1}} d s\right) .
$$

is a fractional derivative and $\left(u_{H-1 / 2} \varphi\right)(s)=s^{H-1 / 2} \varphi(s)$.

We will define a standard cylindrical fractional Brownian motion in a Hilbert space $U$ by the formal series

$$
B^{H}(t):=\sum_{n=1}^{\infty} \beta_{n}^{H}(t) e_{n}
$$

where $\left(e_{n}, n \in \mathbf{N}\right)$ is a complete orthonormal basis in $U$ and $\left(\beta_{n}^{H}(t), n \in \mathbf{N}, t \in \mathbf{R}\right)$ is a sequence of independent, real-valued standard fractional Brownian motions each with the same Hurst parameter $H \in(0,1)$. It is well known that the infinite series (4) does not converge in $L^{2}(\Omega, U)$ so $B^{H}(t)$ is not a well defined $U$-valued random variable. However, it is easy to verify (see [4]) that for any Hilbert space $U_{1}$ such that $U \hookrightarrow U_{1}$ and the embedding is a Hilbert-Schmidt operator, the series (4) defines a $U_{1}$-valued random variable and $\left(B^{H}(t), t \in \mathbf{R}\right)$ is a $U_{1}$-valued fractional Brownian motion of $Q$-covariance type.

Next, the stochastic integral $\int_{0}^{T} G d \beta^{H}$ for an operator-valued function $G:[0, T]$ $\rightarrow \mathcal{L}(U, V)$ is defined.

Definition 2.1. Let $G:[0, T] \rightarrow \mathcal{L}(U, V),\left(e_{n}, n \in \mathbf{N}\right)$ be a complete orthonormal basis in $U, G(\cdot) e_{n} \in \mathcal{H}$ for $n \in \mathbf{N}$, and $B^{H}$ be a standard cylindrical fractional 
Brownian motion in $U$. Let $\beta_{n}^{H}(t):=\left\langle B^{H}(t), e_{n}\right\rangle$ for $n \in \mathbf{N}$. Define

$$
\int_{0}^{T} G d B^{H}:=\sum_{n=1}^{\infty} \int_{0}^{T} G e_{n} d \beta_{n}^{H}
$$

provided the infinite series converges in $L^{2}(\Omega, V)$.

In what follows, it is assumed that $(S(t), t \geq 0)$ is an analytic (and strongly continuous) semigroup on $V$ with infinitesimal generator $A$. In such case, there is a $\hat{\beta} \in \mathbf{R}$ such that the operator $\hat{\beta} I-A$ is uniformly positive on $V$. For each $\delta \geq 0$, let us define $\left(V_{\delta},|\cdot|_{\delta}\right)$ a Hilbert space, where $V_{\delta}=\operatorname{Dom}\left((\hat{\beta} I-A)^{\delta}\right)$ with the graph norm topology such that

$$
|x|_{\delta}=\left|(\hat{\beta} I-A)^{\delta} x\right|_{V} .
$$

The shift $\hat{\beta}$ is fixed. The space $V_{\delta}$ does not depend on $\hat{\beta}$ because the norms are equivalent for different values of $\hat{\beta}$ satisfying the positivity condition.

Consider the linear equation described by a formal stochastic differential

$$
\begin{aligned}
d X(t) & =A X(t) d t+(\hat{\beta} I-A) D d B^{H}(t), \\
X(0) & =x_{0},
\end{aligned}
$$

where $\left(B^{H}(t), t \geq 0\right)$ is a standard cylindrical fractional Brownian motion with Hurst parameter $H \in(0,1)$ in $U$ and $U$ is a separable Hilbert space, $D \in \mathcal{L}\left(U, V_{\varepsilon}\right)$ for a given $\varepsilon>0$ and $x_{0} \in V$ is a random variable.

A solution $\left(X^{x_{0}}(t), t \geq 0\right)$ to $(5)$ is defined in the mild form, i.e.

$$
X^{x_{0}}(t)=S(t) x_{0}+Z(t), \quad t \geq 0
$$

where $(Z(t), t \geq 0)$ is the convolution integral

$$
Z(t)=\int_{0}^{t}(\hat{\beta} I-A) S(t-u) D d B^{H}(u)
$$

Proposition 2.1. Let $H \in(0,1)$. Let $(S(t), t \geq 0)$ be an analytic strongly continuous semigroup such that

$$
|(\hat{\beta} I-A) S(t) D|_{\mathcal{L}_{2}(U, V)} \leq c t^{-\gamma}, \quad t \in(0, T],
$$

for some $T>0, c>0$ and $\gamma \in[0, H)$. Then $(Z(t), t \in[0, T])$ is a well-defined $V_{\delta}$ valued process in $\mathcal{C}^{\beta}\left([0, T], V_{\delta}\right)$, a.s. $\mathbb{P}$ for $\beta+\delta+\gamma<H, \beta \geq 0, \delta \geq 0$. If the semigroup $(S(t), t \geq 0)$ is also exponentially stable, i.e. there exist constants $M>0$ and $\rho>0$ such that for all $t \geq 0$

$$
|S(t)|_{\mathcal{L}(V)} \leq M e^{-\rho t}
$$


then there is a Gaussian centered limiting measure $\mu_{\infty}=\mathcal{N}\left(0, Q_{\infty}\right)$ for $(X(t), t \geq 0)$ such that

$$
\lim _{t \rightarrow \infty} \mu_{t}^{x_{0}}=\mu_{\infty}
$$

for each initial condition $x_{0} \in V$ where $\mu_{t}^{x_{0}}$ is the probability law of $X^{x_{0}}(t)$ and the limit is understood in the sense of weak convergence of measures.

Proof. If $H>1 / 2$, the proof follows from [4], Proposition 3.4 and Corollary 3.5. For $H<1 / 2$, the proof is completely analogous to the proof of Corollary 1.12 and Proposition 1.13 in [6].

Corollary 2.2. Moreover, if we assume that $D \in \mathcal{L}_{2}\left(U, V_{\varepsilon}\right)$, then the condition (A1) is satisfied with $\gamma=1-\varepsilon$.

Proof. The proof follows from the analyticity of the semigroup $(S(t), t \geq 0)$, because

$$
\begin{aligned}
|(\hat{\beta} I-A) S(t) D|_{\mathcal{L}_{2}(U, V)} & \leq\left|(\hat{\beta} I-A)^{1-\varepsilon} S(t)\right|_{\mathcal{L}(V)} \cdot\left|(\hat{\beta} I-A)^{\varepsilon} D\right|_{\mathcal{L}_{2}(U, V)} \\
& \leq c t^{\varepsilon-1}, \quad t \in(0, T] .
\end{aligned}
$$

REMARK 2.1. The covariance $Q_{\infty}$ has for $H>1 / 2$ the following form:

$$
Q_{\infty}=\int_{0}^{\infty} \int_{0}^{\infty}(\hat{\beta} I-A) S(u) D[(\hat{\beta} I-A) S(v) D]^{*} \phi(u-v) d u d v
$$

where $\phi$ is given by

$$
\phi(u)=H(2 H-1)|u|^{2 H-2}
$$

The form for $H<1 / 2$ can be specified in terms of $\mathcal{K}_{H}^{*}$ and a precise statement can be found in [6].

3. Ergodic results. Recall that a measurable $V$-valued process $(X(t), t \geq 0)$ is said to be strictly stationary, if for all $k \in \mathbf{N}$ and for all arbitrary positive numbers $t_{1}, t_{2}, \ldots, t_{k}$, the probability distribution of the $V^{k}$-valued random variable $\left(X\left(t_{1}+\right.\right.$ $\left.r), X\left(t_{2}+r\right), \ldots, X\left(t_{k}+r\right)\right)$ does not depend on $r \geq 0$, i.e.

$$
\operatorname{Law}\left(X\left(t_{1}+r\right), X\left(t_{2}+r\right), \ldots, X\left(t_{k}+r\right)\right)=\operatorname{Law}\left(X\left(t_{1}\right), X\left(t_{2}\right), \ldots, X\left(t_{k}\right)\right)
$$

for all $t_{1}, t_{2}, \ldots, t_{k}, r \geq 0$, where $\operatorname{Law}(\cdot)$ denotes the probability distribution.

Theorem 3.1. If (A1) and (A2) are satisfied, then there exists a strictly stationary solution to $(5)$, i.e. there exists $\tilde{x}$, a random variable on $(\Omega, \mathcal{F}, \mathbb{P})$, such that $\left(X^{\tilde{x}}(t), t \geq 0\right)$ is a strictly stationary process with $\operatorname{Law}\left(X^{\tilde{x}}(t)\right)=\mu_{\infty}, t \geq 0$. In particular $\operatorname{Law}(\tilde{x})=\mu_{\infty}$. 
Proof. The proof follows the lines of an analogous Theorem 3.1 in [12] (where the case of distributed noise is treated). We will only illustrate the main idea.

For $t \geq 0$ let

$$
Y(t):=\int_{-t}^{0}(\hat{\beta} I-A) S(-u) D d B^{H}(u)
$$

It is clear that $Y(t)$ is a $V$-valued random variable on $(\Omega, \mathcal{F}, \mathbb{P})$ with probability law $\mu_{t}^{0}=\mathcal{N}\left(0, Q_{t}\right)$. It must be shown that the limit

$$
\tilde{x}=\lim _{t \rightarrow \infty} Y(t)
$$

exists in $L^{2}(\Omega, V)$ and that

$$
X^{\tilde{x}}(t)=S(t) \tilde{x}+Z(t)
$$

where $Z(t)$ is given by (6), is a stationary solution of (5).

Let $m, n \in \mathbf{N}$. It has to be shown that for an arbitrary increasing sequence of times $\left(t_{n}\right), t_{n} \rightarrow \infty$, the sequence $\left(Y_{t_{n}}\right)$ is a Cauchy sequence in $L^{2}(\Omega, V)$. For all $n \geq m$ we have

$$
\mathbb{E}\left|Y\left(t_{n}\right)-Y\left(t_{m}\right)\right|_{V}^{2}=\mathbb{E}\left|\int_{-t_{n}}^{-t_{m}}(\hat{\beta} I-A) S(-u) D d B^{H}(u)\right|_{V}^{2} .
$$

Denoting by $\tilde{B}^{H}(u)=B^{H}(-u)$ an inverse process that is also a standard cylindrical fractional Brownian motion with stationary increments we have

$$
\mathbb{E}\left|Y\left(t_{n}\right)-Y\left(t_{m}\right)\right|_{V}^{2}=\mathbb{E}\left|\int_{t_{m}}^{t_{n}}(\hat{\beta} I-A) S(u) D d \tilde{B}^{H}(u)\right|_{V}^{2} .
$$

Using the estimate (A1) and following the rest of the proof of the Theorem 3.1 in [12], we can show that the right hand side goes to zero as $m \rightarrow \infty$ and thus $\left(Y\left(t_{n}\right)\right)$ is a Cauchy sequence in $L^{2}(\Omega, V)$. It is easy to see that the limit $\tilde{x}=\lim _{t \rightarrow \infty} Y(t)$ is an initial value yielding the strictly stationary solution.

Theorem 3.2. Let (A1) and (A2) be satisfied and let $\left(X^{\tilde{x}}(t), t \geq 0\right)$ be a strictly stationary solution to (5). Let $\varrho: V \rightarrow \mathbf{R}$ be a measurable functional such that $\mathbb{E}|\varrho(\tilde{x})|<\infty$. Then

$$
\lim _{T \rightarrow \infty} \frac{1}{T} \mathbb{E}\left[\int_{0}^{T} \varrho\left(X^{\tilde{x}}(t)\right) d t\right]=\int_{V} \varrho(y) \mu_{\infty}(d y)
$$


Proof. The proof is obvious, because by Fubini Theorem $\varrho\left(X^{\tilde{x}}(t)\right) \in L^{1}(\Omega \times[0, T])$ and

$$
\begin{aligned}
\lim _{T \rightarrow \infty} \frac{1}{T} \mathbb{E}\left[\int_{0}^{T} \varrho\left(X^{\tilde{x}}(t)\right) d t\right] & =\lim _{T \rightarrow \infty} \frac{1}{T} \int_{0}^{T} \mathbb{E}\left[\varrho\left(X^{\tilde{x}}(t)\right)\right] d t \\
& =\lim _{T \rightarrow \infty} \frac{1}{T} \int_{0}^{T} \mathbb{E}[\varrho(\tilde{x})] d t \\
& =\mathbb{E}[\varrho(\tilde{x})] \\
& =\int_{V} \varrho(y) \mu_{\infty}(d y) .
\end{aligned}
$$

If the initial condition is arbitrary, the following result may be useful:

Theorem 3.3. Let (A1) and (A2) be satisfied and let $\left(X^{x_{0}}(t), t \geq 0\right)$ be a solution to (5) with initial condition $X(0)=x_{0} \in V$, a random variable. Let $\varrho: V \rightarrow \mathbf{R}$ be a functional satisfying the following local Lipschitz condition: let there exist real constants $K>0$ and $m \geq 1$ such that

$$
|\varrho(x)-\varrho(y)| \leq K|x-y|_{V}\left(1+|x|_{V}^{m}+|y|_{V}^{m}\right)
$$

for all $x, y \in V$. Assume also that

$$
\mathbb{E}\left|x_{0}\right|_{V}^{2 m}<\infty
$$

Then

$$
\lim _{T \rightarrow \infty} \frac{1}{T} \mathbb{E} \int_{0}^{T} \varrho\left(X^{x_{0}}(t)\right) d t=\int_{V} \varrho(y) \mu_{\infty}(d y)
$$

Proof. The desired convergence can be rewritten as

$$
\lim _{T \rightarrow \infty}\left|\frac{1}{T} \mathbb{E} \int_{0}^{T} \varrho\left(X^{x_{0}}(t)\right) d t-\int_{V} \varrho(y) \mu_{\infty}(d y)\right|=0 .
$$

Let $\left(X^{\tilde{x}}(t), t \geq 0\right)$ be a strictly stationary solution to (5). Then obviously

$$
\begin{aligned}
\mid \frac{1}{T} \mathbb{E} \int_{0}^{T} \varrho\left(X^{x_{0}}(t)\right) d t & -\frac{1}{T} \mathbb{E} \int_{0}^{T} \varrho\left(X^{\tilde{x}}(t)\right) d t \mid \\
& \leq \frac{1}{T} \mathbb{E} \int_{0}^{T}\left|\varrho\left(X^{x_{0}}(t)\right)-\varrho\left(X^{\tilde{x}}(t)\right)\right| d t
\end{aligned}
$$

We will show that the right hand side of (10) goes to zero as $T \rightarrow \infty$. Using the local 
Lipschitz assumption (8) we get

$$
\begin{aligned}
\frac{1}{T} & \mathbb{E} \int_{0}^{T}\left|\varrho\left(X^{x_{0}}(t)\right)-\varrho\left(X^{\tilde{x}}(t)\right)\right| d t \\
& \leq \frac{K}{T} \mathbb{E} \int_{0}^{T}\left|X^{x_{0}}(t)-X^{\tilde{x}}(t)\right|_{V}\left(1+\left|X^{x_{0}}(t)\right|_{V}^{m}+\left|X^{\tilde{x}}(t)\right|_{V}^{m}\right) d t \\
& =\frac{K}{T} \int_{0}^{T} \mathbb{E}\left|S(t)\left(x_{0}-\tilde{x}\right)\right|_{V}\left(1+\left|X^{x_{0}}(t)\right|_{V}^{m}+\left|X^{\tilde{x}}(t)\right|_{V}^{m}\right) d t \\
& \leq \frac{K}{T} \int_{0}^{T}|S(t)|_{\mathcal{L}(V)} \mathbb{E}\left|\left(x_{0}-\tilde{x}\right)\right|_{V}\left(1+\left|X^{x_{0}}(t)\right|_{V}^{m}+\left|X^{\tilde{x}}(t)\right|_{V}^{m}\right) d t
\end{aligned}
$$

in virtue of the exponential stability bound (A2)

$$
\leq \frac{K M}{T} \int_{0}^{T} e^{-\rho t} \mathbb{E}\left|x_{0}-\tilde{x}\right|_{V}\left(1+\left|X^{x_{0}}(t)\right|_{V}^{m}+\left|X^{\tilde{x}}(t)\right|_{V}^{m}\right) d t
$$

and by the Hölder inequality

$$
\leq \frac{K M}{T} \int_{0}^{T} e^{-\rho t}\left(\mathbb{E}\left|x_{0}-\tilde{x}\right|_{V}^{2}\right)^{1 / 2}\left(\mathbb{E}\left(1+\left|X^{x_{0}}(t)\right|_{V}^{m}+\left|X^{\tilde{x}}(t)\right|_{V}^{m}\right)^{2}\right)^{1 / 2} d t .
$$

To show that the last term on the right hand side goes to 0 as $T \rightarrow \infty$, we have to show that

1. $\sup _{t \in \mathbf{R}_{+}} \mathbb{E}\left|X^{\tilde{x}}(t)\right|^{2 m}<\infty$. But this is equivalent to $\mathbb{E}|\tilde{x}|^{2 m}<\infty$, which is satisfied, because $\tilde{x}$ is Gaussian with the probability distribution $\mu_{\infty}=$ $\mathcal{N}\left(0, Q_{\infty}\right)$ and all Gaussian measures have all their moments finite.

2. $\sup _{t \in \mathbf{R}_{+}} \mathbb{E}\left|X^{x_{0}}(t)\right|^{2 m}<\infty$. Let $Z(t)=\int_{0}^{t}(\hat{\beta} I-A) S(t-u) D d B^{H}(u)$. Then

$$
\begin{aligned}
\sup _{t \in \mathbf{R}_{+}} \mathbb{E}\left|X^{x_{0}}(t)\right|^{2 m} & =\sup _{t \in \mathbf{R}_{+}} \mathbb{E}\left|S(t) x_{0}+Z(t)\right|^{2 m} \\
& \leq c_{m}\left(\sup _{t \in \mathbf{R}_{+}} \mathbb{E}\left|S(t) x_{0}\right|^{2 m}+\sup _{t \in \mathbf{R}_{+}} \mathbb{E}|Z(t)|^{2 m}\right) .
\end{aligned}
$$

By the exponential stability bound (A2) for the first term and the fact that $Z(t)$ is Gaussian with probability distribution $\mathcal{N}\left(0, Q_{t}\right)$

$$
\begin{aligned}
\leq & c_{m} \sup _{t \in \mathbf{R}_{+}} M e^{-2 m \rho t} \mathbb{E}\left|x_{0}\right|^{2 m}+c_{m} \sup _{t \in \mathbf{R}_{+}}\left(\operatorname{Tr} Q_{t}\right)^{m} \\
\leq & c_{1} \mathbb{E}\left|x_{0}\right|^{2 m} \\
& +c_{m} \sup _{t \in \mathbf{R}_{+}}\left(\int_{0}^{t} \int_{0}^{t}|S(u) \Phi|_{\mathcal{L}_{2}(U, V)}|S(v) \Phi|_{\mathcal{L}_{2}(U, V)} \phi(u-v) d u d v\right)^{m}
\end{aligned}
$$

and following the steps in the proof of Theorem 3.1 for $m=0$ and $n=t$ we deduce that the last term on the right hand side is bounded. 
We showed that the right hand side of (10) goes to zero as $T \rightarrow \infty$, which yields (9) using the Theorem 3.2 and completes the proof. Note that $\rho$ satisfied the integrability condition from Theorem 3.2 because by (8) it has at most polynomial growth and $\tilde{x}$ is a Gaussian random variable.

Now we focus our attention on the pathwise ergodic behaviour. At first we recall the famous Birkhoff's theorem for strictly stationary processes.

Theorem 3.4 (Birkhoff's theorem). Let $\left(X^{\tilde{x}}(t), t \geq 0\right)$ be a $V$-valued strictly stationary process on $(\Omega, \mathcal{F}, \mathbb{P})$. Then for all measurable functionals $\varrho: V \rightarrow \mathbf{R}$ such that $\mathbb{E}|\varrho(\tilde{x})|<\infty$ there exists a measurable functional $\xi: \Omega \rightarrow \mathbf{R}$ such that

$$
\lim _{T \rightarrow \infty} \frac{1}{T} \int_{0}^{T} \varrho\left(X_{t}^{\tilde{x}}\right) d t=\xi, \quad \text { a.s.-P. }
$$

Proof. See e.g [21].

Recall that a $V$-valued strictly stationary process $(X(t), t \geq 0)$ is said to be ergodic, if $\xi$ in (11) does not depend on $\omega \in \Omega$, i.e. $\xi$ is deterministic, and $\xi=\mathbb{E}[\varrho(\tilde{x})]$.

Theorem 3.5 (Ergodic theorem for a strictly stationary solution). Let $\left(X^{\tilde{x}}(t)\right.$, $t \geq 0$ ) be a $V$-valued strictly stationary solution to (5). Let $\varrho: V \rightarrow \mathbf{R}$ be a measurable functional such that $\mathbb{E}|\varrho(\tilde{x})|<\infty$. Then

$$
\lim _{T \rightarrow \infty} \frac{1}{T} \int_{0}^{T} \varrho\left(X^{\tilde{x}}(t)\right) d t=\int_{V} \varrho(y) \mu_{\infty}(d y), \quad \text { a.s. }-\mathbb{P} .
$$

Proof. Let $z \in V$ be arbitrary and let $(Y(t), t \geq 0)$ be a $\mathbf{R}$-valued process defined by

$$
Y(t):=\left\langle X^{\tilde{x}}(t), z\right\rangle_{V}
$$

Then $Y(0)=\langle\tilde{x}, z\rangle_{V}$. The process $(Y(t), t \geq 0)$ is a $\mathbf{R}$-valued strictly stationary centered Gaussian process. Let $R(t):=\mathbb{E}[Y(0) Y(t)]$ be the correlation function of the process $Y(t)$. Then (cf. [21]) the process $Y(t)$ is ergodic if $\lim _{t \rightarrow \infty} R(t)=0$. We have

$$
\begin{aligned}
R(t) & =\mathbb{E}[Y(0) Y(t)] \\
& =\mathbb{E}\left[\langle\tilde{x}, z\rangle_{V}\left\langle X^{\tilde{x}}(t), z\right\rangle_{V}\right] \\
& =\mathbb{E}\left[\langle\tilde{x}, z\rangle_{V}\left\langle S(t)(\tilde{x})+\int_{0}^{t}(\hat{\beta} I-A) S(t-r) D d B^{H}(r), z\right\rangle_{V}\right] \\
& =\mathbb{E}[\underbrace{\left[\langle\tilde{x}, z\rangle_{V}\langle S(t)(\tilde{x}), z\rangle_{V}\right]}_{I}+\mathbb{E} \underbrace{\left[\langle\tilde{x}, z\rangle_{V}\left\langle\int_{0}^{t}(\hat{\beta} I-A) S(t-r) D d B^{H}(r), z\right\rangle_{V}\right]}_{I I} .
\end{aligned}
$$

We will estimate both terms separately. 
The first term

$$
\begin{aligned}
\mathbb{E}|I| & =\mathbb{E}\left|\langle\tilde{x}, z\rangle_{V}\langle S(t)(\tilde{x}), z\rangle_{V}\right| \\
& \leq \mathbb{E}|S(t) \tilde{x}|_{V}|z|_{V}^{2}|\tilde{x}|_{V}
\end{aligned}
$$

and using the exponential stability (A2)

$$
\leq M e^{-\rho t}|z|_{V}^{2} \mathbb{E}|\tilde{x}|_{V}^{2}
$$

which goes to 0 as $t \rightarrow \infty$, because $\mathbb{E}|\tilde{x}|_{V}^{2}<\infty$. The second term is for $H=1 / 2$ equal to zero, because $\tilde{x}$ and the convolution integral $\int_{0}^{t}(\hat{\beta} I-A) S(t-r) D d B^{H}(r)$ are stochastically independent and therefore

$$
\begin{aligned}
\mathbb{E}[I I] & =\mathbb{E}\left[\langle\tilde{x}, z\rangle_{V}\left\langle\int_{0}^{t}(\hat{\beta} I-A) S(t-r) D d B^{H}(r), z\right\rangle_{V}\right] \\
& =\mathbb{E}\left[\langle\tilde{x}, z\rangle_{V}\right] \mathbb{E}\left[\left\langle\int_{0}^{t}(\hat{\beta} I-A) S(t-r) D d B^{H}(r), z\right\rangle_{V}\right] \\
& =0 .
\end{aligned}
$$

For $H \neq 1 / 2$ we obtain that $\mathbb{E}[I I]$ goes to zero as $t \rightarrow \infty$ by following Lemmas 4.4 and 4.5 from [12]. Thus the process $Y(t)=\left\langle X^{\tilde{x}}(t), z\right\rangle_{V}$ is ergodic for each $z \in V$.

Take $\left(h_{n}, n \in \mathbf{N}\right)$ any orthonormal basis in $V$. Then

$$
\mathbb{E}\left\langle\tilde{x}, h_{n}\right\rangle_{V}=\lim _{T \rightarrow \infty} \frac{1}{T} \int_{0}^{T}\left\langle X^{\tilde{x}}(t), h_{n}\right\rangle_{V} d t=0
$$

on $\Omega_{n} \subset \Omega, \mathbb{P}\left(\Omega_{n}\right)=1$. On the other hand, by Theorem 3.4

$$
\lim _{T \rightarrow \infty} \frac{1}{T} \int_{0}^{T} X^{\tilde{x}}(t) d t=\xi
$$

on $\Omega_{0} \subset \Omega, \mathbb{P}\left(\Omega_{0}\right)=1$. Taking $\Omega^{\prime}=\bigcap_{n=0}^{\infty} \Omega_{n}$, we have $\mathbb{P}\left(\Omega^{\prime}\right)=1$ and

$$
\begin{aligned}
0 & =\lim _{T \rightarrow \infty} \frac{1}{T} \int_{0}^{T}\left\langle X^{\tilde{x}}(t), h_{n}\right\rangle_{V} d t \\
& =\lim _{T \rightarrow \infty}\left\langle\frac{1}{T} \int_{0}^{T} X^{\tilde{x}}(t) d t, h_{n}\right\rangle_{V} \\
& =\left\langle\lim _{T \rightarrow \infty} \frac{1}{T} \int_{0}^{T} X^{\tilde{x}}(t) d t, h_{n}\right\rangle_{V} \\
& =\left\langle\xi, e_{n}\right\rangle_{V}
\end{aligned}
$$

on $\Omega^{\prime}$. Hence $\left\langle\xi, h_{n}\right\rangle_{V}=0$ for each $n$ on $\Omega^{\prime}$, i.e. $\xi=0$, a.s.-P, and it follows that $X^{\tilde{x}}(t)$ is ergodic. 
We will now apply the previous results to the solution of (5) with arbitrary initial condition.

Definition 3.1. A $V$-valued Gaussian process $\left(B_{Q}^{H}(t), t \in \mathbf{R}\right)$ on $(\Omega, \mathcal{F}, \mathbb{P})$ is called a fractional Brownian motion of Q-covariance type with Hurst parameter $H \in(0,1)$ (or, more simply, a fractional Q-Brownian motion with Hurst parameter $H)$ if

1. $\mathbb{E} B_{Q}^{H}(t)=0$ for all $t \in \mathbf{R}$,

2. $\operatorname{Cov}\left(B_{Q}^{H}(t), B_{Q}^{H}(s)\right)=\frac{1}{2}\left(|t|^{2 H}+|s|^{2 H}-|t-s|^{2 H}\right) Q$, for all $s, t \in \mathbf{R}$,

3. $\left(B_{Q}^{H}(t), t \in \mathbf{R}\right)$ has $V$-valued, continuous sample paths a.s. $\mathbb{P}$.

Let $\left(B^{H}(t), t \in \mathbf{R}\right)$ be a standard cylindrical fractional Brownian motion in $U$. Let $\Phi \in \mathcal{L}_{2}(U, V)$ and set $Q=\Phi \Phi^{*}$. Then there exists (see e.g. [15], Proposition 1.1.1) a $Q$-covariance fractional Brownian motion $\left(B_{Q}^{H}(t), t \in \mathbf{R}\right)$ such that for all $z \in V$

$$
\left\langle B_{Q}^{H}(t), z\right\rangle_{V}=\left\langle B^{H}(t), \Phi^{*} z\right\rangle, \quad \text { a.s.- } \mathbb{P} .
$$

Moreover, the convolution integral $\int_{0}^{t} S(t-r) \Phi d B^{H}(r)$ solves the equation

$$
\begin{aligned}
d X(t) & =A X(t) d t+d B_{Q}^{H}(t), \\
X(0) & =0 .
\end{aligned}
$$

We recall some a.s.- $\mathbb{P}$ growth estimates and a representation of the solution to equation (12) that have been proved in [13].

Lemma 3.6. Let $\Phi \in \mathcal{L}_{2}(U, V)$. Then

$$
\int_{0}^{t} S(t-r) d B_{Q}^{H}(r)=A \int_{0}^{t} S(t-r) B_{Q}^{H}(r) d r+B_{Q}^{H}(t), \quad \text { a.s.- } \mathbb{P},
$$

for $t \geq 0$. Moreover, for any $\omega \in \Omega, \varepsilon>0$ there exists a constant $k(\omega, \varepsilon)$ such that

$$
\left|B_{Q}^{H}(t)\right|_{V} \leq \varepsilon t^{2}+k(\omega, \varepsilon)
$$

for $t \in \mathbf{R}$.

Proof. Follows from [13] Lemmas 2.4 and 2.6.

Theorem 3.7. Let (A1) and (A2) be satisfied and let $\left(X^{x_{0}}(t), t \geq 0\right)$ be a solution to (5) with $D \in \mathcal{L}_{2}(U, V)$. Let $\varphi: \mathbf{R} \rightarrow \mathbf{R}$ be a real function satisfying the following local Lipschitz condition: let there exist real constants $K>0$ and $m \geq 1$ such that

$$
|\varphi(x)-\varphi(y)| \leq K|x-y|\left(1+|x|^{m}+|y|^{m}\right)
$$

for all $x, y \in \mathbf{R}$. Let $z \in \operatorname{Dom}\left(\left(A^{*}\right)^{2}\right)$ be arbitrary. Then

$$
\lim _{T \rightarrow \infty} \frac{1}{T} \int_{0}^{T} \varphi\left(\left\langle X^{x_{0}}(t), z\right\rangle\right) d t=\int_{V} \varphi(y) \mu_{\infty}(d y), \quad \text { a.s. }-\mathbb{P} .
$$


Proof. Let $\left(X^{\tilde{x}}(t), t \geq 0\right)$ be a strictly stationary solution to (5). Then obviously

$$
\begin{aligned}
\mid \frac{1}{T} \int_{0}^{T} \varphi\left(\left\langle X^{x_{0}}(t), z\right\rangle_{V}\right) d t & -\frac{1}{T} \int_{0}^{T} \varphi\left(\left\langle X^{\tilde{x}}(t), z\right\rangle_{V}\right) d t \mid \\
& \leq \frac{1}{T} \int_{0}^{T}\left|\varphi\left(\left\langle X^{x_{0}}(t), z\right\rangle_{V}\right)-\varphi\left(\left\langle X^{\tilde{x}}(t), z\right\rangle_{V}\right)\right| d t .
\end{aligned}
$$

We will show that the right hand side goes to zero as $T \rightarrow \infty$. Using the local Lipschitz assumption (13) we get

$$
\begin{aligned}
& \frac{1}{T} \int_{0}^{T}\left|\varphi\left(\left\langle X^{x_{0}}(t), z\right\rangle_{V}\right)-\varphi\left(\left\langle X^{\tilde{x}}(t), z\right\rangle_{V}\right)\right| d t \\
& \leq \frac{K}{T} \int_{0}^{T}\left|\left\langle X^{x_{0}}(t)-X^{\tilde{x}}(t), z\right\rangle_{V}\right|\left(1+\left|\left\langle X^{x_{0}}(t), z\right\rangle_{V}\right|^{m}+\left|\left\langle X^{\tilde{x}}(t), z\right\rangle_{V}\right|^{m}\right) d t \\
& \leq \frac{K}{T} \int_{0}^{T}\left|\left\langle S(t)\left(x_{0}-\tilde{x}\right), z\right\rangle_{V}\right| \\
& \quad \cdot\left(1+\left|\left\langle S(t) x_{0}+Z(t), z\right\rangle_{V}\right|^{m}+\left|\langle S(t) \tilde{x}+Z(t), z\rangle_{V}\right|^{m}\right) d t \\
& \leq \frac{K}{T} \int_{0}^{T}|S(t)|_{\mathcal{L}(V)}\left|x_{0}-\tilde{x}\right|_{V}|z|_{V} \\
& \quad \cdot\left(1+c_{1}|S(t)|_{\mathcal{L}(V)}^{m}\left(\left|x_{0}\right|_{V}^{m}+|\tilde{x}|_{V}^{m}\right)+c_{2}\left|\langle Z(t), z\rangle_{V}\right|^{m}\right) d t
\end{aligned}
$$

and in virtue of the exponential stability bound (A2)

$$
\begin{aligned}
\leq & \frac{K M}{T}\left|x_{0}-\tilde{x}\right|_{V}|z|_{V} \\
& \cdot \int_{0}^{T} e^{-\rho t}\left(1+c_{1} M e^{-\rho m t}\left(\left|x_{0}\right|_{V}^{m}+|\tilde{x}|_{V}^{m}\right)+c_{2}\left|\langle Z(t), z\rangle_{V}\right|^{m}\right) d t \\
(15) \leq & \frac{c_{3}}{T} \int_{0}^{T} e^{-\rho t}\left(1+c_{4} e^{-\rho m t}+c_{2}\left|\langle Z(t), z\rangle_{V}\right|^{m}\right) d t .
\end{aligned}
$$

We need the last term on the right hand side to go to 0 as $T \rightarrow \infty$. Applying Lemma 3.6 with $\Phi=D$ we have

$$
\begin{aligned}
\left|\langle Z(t), z\rangle_{V}\right|= & \left|\left\langle(\hat{\beta} I-A) \int_{0}^{t} S(t-r) D d B^{H}(r), z\right\rangle_{V}\right| \\
= & \left|\left\langle\int_{0}^{t} S(t-r) D d B^{H}(r),(\hat{\beta} I-A)^{*} z\right\rangle_{V}\right| \\
\leq & \left|\left\langle\int_{0}^{t} S(t-r) B_{Q}^{H}(r) d r, A^{*}\left(\hat{\beta} I-A^{*}\right) z\right\rangle_{V}+\left\langle B_{Q}^{H}(t),\left(\hat{\beta} I-A^{*}\right) z\right\rangle_{V}\right| \\
\leq & \left|A^{*}\left(\hat{\beta} I-A^{*}\right) z\right|_{V} \\
& \quad \cdot \int_{0}^{t}|S(t-r)|_{\mathcal{L}(V)}\left|B_{Q}^{H}(r)\right|_{V} d r+\left|B_{Q}^{H}(t)\right|_{V}\left|\left(\hat{\beta} I-A^{*}\right) z\right|_{V}
\end{aligned}
$$


and by the exponential stability (A2) and the bound from Lemma 3.6

$$
\begin{aligned}
& \leq M\left|A^{*}\left(\hat{\beta} I-A^{*}\right) z\right|_{V} \\
& \quad \cdot \int_{0}^{t} e^{-\rho(t-r)}\left(\varepsilon r^{2}+k(\omega, \varepsilon)\right) d r+\left(\varepsilon t^{2}+k(\omega, \varepsilon)\right)\left|\left(\hat{\beta} I-A^{*}\right) z\right|_{V} \\
& \leq \tilde{c}(\omega)\left(1+t^{2}\right) .
\end{aligned}
$$

Therefore

$$
(15) \leq \frac{c(\omega)}{T} \int_{0}^{T} e^{-\rho m t}\left(1+t^{2}\right)^{m} d t
$$

which goes to zero as $T \rightarrow \infty$, which yields (14) using Theorem 3.5 and thus completes the proof.

4. Parameter estimates based on ergodicity. In this section we present the results on parameter estimation in infinite dimensional equations that are based on the ergodic theorems proved in the previous section.

Consider the linear equation

$$
\begin{aligned}
d X(t) & =\alpha A X(t) d t+\alpha(\hat{\beta} I-A) D d B^{H}(t), \\
X(0) & =x_{0},
\end{aligned}
$$

where $\alpha>0$ is a real constant parameter, $A, \hat{\beta}, B^{H}, D$ and $x_{0}$ are as in (5) and, moreover, $D \in \mathcal{L}_{2}(U, V)$.

The operator $\alpha A$ is the infinitesimal generator of the semigroup $\left(S_{\alpha}(t), t \geq 0\right)$. Obviously $S_{\alpha}(t)=S(\alpha t)$ for all $t \geq 0$. The semigroup $\left(S_{\alpha}(t), t \geq 0\right)$ is analytic and the operator $\alpha \hat{\beta} I-\alpha A$ is positive. The semigroup $\left(S_{\alpha}(t), t \geq 0\right)$ is also exponentially stable and the limiting measure has the form $\mu_{\infty}^{\alpha}=\mathcal{N}\left(0, Q_{\infty}^{\alpha}\right)$.

For $H>1 / 2$ we have (cf. Remark 2.1)

$$
\begin{aligned}
Q_{\infty}^{\alpha} & =\int_{0}^{\infty} \int_{0}^{\infty} \alpha(\hat{\beta} I-A) S_{\alpha}(u) D\left[\alpha(\hat{\beta} I-A) S_{\alpha}(v) D\right]^{*} \phi(u-v) d u d v \\
& =\int_{0}^{\infty} \int_{0}^{\infty}(\hat{\beta} I-A) S(u) D[(\hat{\beta} I-A) S(v) D]^{*} \phi\left(\frac{u}{\alpha}-\frac{v}{\alpha}\right) d u d v \\
& =\alpha^{2-2 H} \int_{0}^{\infty} \int_{0}^{\infty}(\hat{\beta} I-A) S(u) D[(\hat{\beta} I-A) S(v) D]^{*} \phi(u-v) d u d v \\
& =\alpha^{2-2 H} Q_{\infty}
\end{aligned}
$$

where $Q_{\infty}$ corresponds to the case $\alpha=1$. 
For $H<1 / 2$ and $x, y \in V$ we have

$$
\begin{aligned}
&\left\langle Q_{\infty}^{\alpha} x, y\right\rangle_{V} \\
&=\lim _{T \rightarrow \infty}\left\langle Q_{T}^{\alpha} x, y\right\rangle_{V} \\
&=\lim _{T \rightarrow \infty} \sum_{n=1}^{\infty} \mathbb{E}\left\langle\int_{0}^{T} \alpha(\hat{\beta} I-A) S_{\alpha}(r) D h_{n} d \beta_{n}^{H}(r), x\right\rangle_{V} \\
& \cdot\left\langle\int_{0}^{T} \alpha(\hat{\beta} I-A) S_{\alpha}(r) D h_{n} d \beta_{n}^{H}(r), y\right\rangle_{V} \\
&=\lim _{T \rightarrow \infty} \alpha^{2} \int_{0}^{T} \sum_{n=1}^{\infty}\left\langle\mathcal{K}_{H}^{*}\left((\hat{\beta} I-A) S_{\alpha}(\cdot) D h_{n}\right)(r), x\right\rangle_{V} \\
& \cdot\left\langle\mathcal{K}_{H}^{*}(\hat{\beta} I-A)\left(S_{\alpha}(\cdot) D h_{n}\right)(r), x\right\rangle_{V} d r .
\end{aligned}
$$

Using the representation (3) and a simple substitution theorem we also arrive at

$$
\left\langle Q_{\infty}^{\alpha} x, y\right\rangle_{V}=\alpha^{2-2 H}\left\langle Q_{\infty} x, y\right\rangle_{V}
$$

for all $x, y \in V$ and therefore

$$
Q_{\infty}^{\alpha}=\alpha^{2-2 H} Q_{\infty}
$$

For $H=1 / 2$ this equality is obvious.

Now we define a family of estimators of the parameter $\alpha$.

Theorem 4.1. Let (A1) and (A2) be satisfied and let $\left(X^{x_{0}}(t), t \geq 0\right)$ be a $V$ valued solution to (16) with $D \in \mathcal{L}_{2}(U, V)$. Let $z \in \operatorname{Dom}\left(\left(A^{*}\right)^{2}\right)$ be arbitrary such that

$$
\left\langle Q_{\infty} z, z\right\rangle_{V}>0
$$

Define

$$
\hat{\alpha}_{T}:=\left(\frac{\left\langle Q_{\infty} z, z\right\rangle_{V}}{\frac{1}{T} \int_{0}^{T}\left|\left\langle X^{x_{0}}(t), z\right\rangle_{V}\right|^{2} d t}\right)^{\frac{1}{2 H-2}} .
$$

Then

$$
\lim _{T \rightarrow \infty} \hat{\alpha}_{T}=\alpha, \quad \text { a.s. }-\mathbb{P},
$$

i.e., $\left(\hat{\alpha}_{T}\right)$ is a strongly consistent family of estimators.

Proof. Let $z \in \operatorname{Dom}\left(\left(A^{*}\right)^{2}\right)$ be arbitrary. Let $\varphi: \mathbf{R} \rightarrow \mathbf{R}, \varphi(y)=|y|^{2}$. Then all 
the conditions of Theorem 3.7 are satisfied with $m=1$ and

$$
\begin{aligned}
\lim _{T \rightarrow \infty} \frac{1}{T} \int_{0}^{T} \varphi\left(\left\langle X^{x_{0}}(t), z\right\rangle_{V}\right) d t & =\lim _{T \rightarrow \infty} \frac{1}{T} \int_{0}^{T}\left|\left\langle X^{x_{0}}(t), z\right\rangle_{V}\right|^{2} d t \\
& =\int_{V}\left|\langle y, z\rangle_{V}\right|^{2} \mu_{\infty}(d y) \\
& =\left\langle Q_{\infty}^{\alpha} z, z\right\rangle_{V} \\
& =\alpha^{2-2 H}\left\langle Q_{\infty} z, z\right\rangle_{V}, \quad \text { a.s.-P }
\end{aligned}
$$

which completes the proof.

REMARK 4.1. If the initial condition $x_{0} \in V$ is such that $\mathbb{E}\left|x_{0}\right|_{V}^{2}<\infty$ and if the limiting measure $\mu_{\infty}$ exists with covariance $Q_{\infty}$ such that $\operatorname{Tr} Q_{\infty} \neq 0$, we can define

$$
\hat{\alpha}_{T}:=\left(\frac{\operatorname{Tr} Q_{\infty}}{\frac{1}{T} \mathbb{E} \int_{0}^{T}\left|X^{x_{0}}(t)\right|_{V}^{2} d t}\right)^{\frac{1}{2 H-2}} .
$$

Then we can use the Theorem 3.3 with $\varrho: V \rightarrow \mathbf{R}, \varrho(y)=|y|_{V}^{2}, y \in V$ and $m=1$ to show that

$$
\lim _{T \rightarrow \infty} \hat{\alpha}_{T}=\alpha .
$$

Some examples of parameter estimations including numerical simulations can be found in [16] and [17].

5. Example. Now we will apply the above obtained results to the heat equation formally described as

$$
\frac{\partial y}{\partial t}=\alpha(\Delta-\delta I) y(t, \xi), \quad(t, \xi) \in \mathbf{R}_{+} \times \mathcal{O},
$$

where $\Delta$ denotes the Laplace operator, $\alpha>0$ is an unknown parameter, $\delta \geq 0$ is fixed and $\mathcal{O} \subset \mathbf{R}^{d}$ is a bounded domain with smooth boundary $\partial \mathcal{O}$. The equation (17) is considered together with an initial condition

$$
y(0, \xi)=x_{0}(\xi), \quad \xi \in \mathcal{O}
$$

and boundary condition either of Dirichlet type

$$
y(t, \xi)=\eta^{H}(t, \xi), \quad(t, \xi) \in \mathbf{R}_{+} \times \partial \mathcal{O},
$$

or of Neumann type

$$
\frac{\partial y}{\partial \nu}(t, \xi)=\eta^{H}(t, \xi), \quad(t, \xi) \in \mathbf{R}_{+} \times \partial \mathcal{O},
$$

where $\nu$ is the normal derivative and $\eta^{H}$ stands formally for a Gaussian noise, fractional in time. 
The problem (17),(18),(19) or (17),(18),(20) may be rewritten in the form (16) with $V=L^{2}(\mathcal{O}), U=L^{2}(\partial \mathcal{O})$ (if the underlying space dimension $d=1, U=\mathbf{R}^{2}$ ), $A=\left.(\Delta-\delta I)\right|_{\operatorname{Dom}(A)}$ with $\operatorname{Dom}(A)=H^{2}(\mathcal{O}) \cap H_{0}^{1}(\mathcal{O})$ (in the case of Dirichlet boundary conditions) or $\operatorname{Dom}(A)=\left\{\varphi \in H^{2}(\mathcal{O}) ; \frac{\partial \varphi}{\partial \nu}=0\right.$ on $\left.\partial \mathcal{O}\right\}$ (for the Neumann boundary conditions). Furthermore, $\left(B^{H}(t), t \geq 0\right)$ is a cylindrical fractional Brownian motion process on $U$ (if $d=1, B^{H}(t)$ is just two-dimensional), $x_{0} \in V \in L^{2}(\mathcal{O})$ and $D=N Q^{1 / 2}$, where $Q^{1 / 2} \in \mathcal{L}(U)$ is the covariance operator of the noise and $N$ stands for the Dirichlet or Neumann map obtained in the following way:

Take an arbitrary $\hat{\beta} \geq 0$ such that $\hat{\beta} I-A$ is uniformly positive (we may take $\hat{\beta}=0$ in the case of Dirichlet boundary condition and any $\hat{\beta}>0$ for the Neumann case) and consider the elliptic equation

$$
\begin{aligned}
&(\hat{\beta} I-A) z=0 \text { in } \quad \mathcal{O}, \\
& z=h \quad \text { on } \quad \partial \mathcal{O},
\end{aligned}
$$

where $h \in U$. The mapping $N$ is defined as $N(h)=z$.

The equation (16) is a version of the problem (17),(18),(19) or (17),(18),(20) (cf. [4]; for $H=1 / 2$, when $B^{H}$ is a standard Wiener process, this interpretation - for a fixed parameter value - has been used in numerous papers, see the monograph [2], Chapter 13 and the references therein). As we need justify the special form of the dependence on the parameter, we will sketch the main argument. Let $u(t)$ be a two times continuously differentiable function with values in $U$ such that $x_{0}-N u(0) \in$ $\operatorname{Dom}(A)$. Then there exists a strong solution to the equation

$$
\begin{aligned}
& z^{\prime}(t)=\alpha A z(t)+\alpha \hat{\beta} N u(t)-N u^{\prime}(t), \quad t>0, \\
& z(0)=x_{0}-N u(0) .
\end{aligned}
$$

Set $v(t)=z(t)+N u(t)$. Then $v(0)=z(0)+N u(0)$ and

$$
\begin{aligned}
\frac{d}{d t} v(t) & =\frac{d}{d t}(z(t)+N u(t)) \\
& =z^{\prime}(t)+N u^{\prime}(t) \\
& =\alpha A z(t)+\alpha \hat{\beta} N u(t) \\
& =\alpha A(v(t)-N u(t))+\alpha \hat{\beta} N u(t) \\
& =\alpha A v(t)+\alpha(\hat{\beta} I-A) N u(t) \\
& =\alpha A v(t)
\end{aligned}
$$

because $\alpha(\hat{\beta} I-A) N u(t)=0$ for each $t>0$. Since $z$ (being a strong solution to $(21),(22))$ satisfies the zero boundary condition (in either Dirichlet or Neumann case), we have

$$
\left.v\right|_{\mathbf{R}_{+} \times \partial \mathcal{O}}=u \quad \text { or }\left.\quad \frac{\partial v}{\partial \nu}\right|_{\mathbf{R}_{+} \times \partial \mathcal{O}}=u
$$


Therefore, $v(t)$ is the solution to the problem

$$
\begin{aligned}
\frac{d}{d t} v(t) & =\alpha A v(t), \quad t>0, \\
v(0) & =x_{0},
\end{aligned}
$$

with inhomogeneous boundary condition (23). Now we will find the mild form of the solution to $v$. The mild form of the solution $z(t)$ to $(21),(22)$ is

$$
z(t)=S_{\alpha}(t)\left(x_{0}-N u(0)\right)+\int_{0}^{t} S_{\alpha}(t-s)\left(\alpha \hat{\beta} N u(s)-N u^{\prime}(s)\right) d s, \quad t>0,
$$

where $S_{\alpha}(t)=S(\alpha t), t \geq 0$ and $(S(t), t \geq 0)$ is the semigroup generated by $A$. Thus

$v(t)=z(t)+N u(t)=S_{\alpha}(t)\left(x_{0}-N u(0)\right)+\int_{0}^{t} S_{\alpha}(t-s)\left(\alpha \hat{\beta} N u(s)-N u^{\prime}(s)\right) d s+N u(t)$,

and since the integration by parts yields

$$
\int_{0}^{t} S_{\alpha}(t-s) N u^{\prime}(s) d s=N u(t)-S_{\alpha}(t) N u(0)+\alpha \int_{0}^{t} A S_{\alpha}(t-s) N u(s) d s,
$$

it follows that

$$
v(t)=S_{\alpha}(t) x_{0}+\alpha(\hat{\beta} I-A) \int_{0}^{t} S_{\alpha}(t-s) N u(s) d s, \quad t>0 .
$$

Similarly as in the case of distributed noise, the mild solution of our problem (16) is motivated by (24) where $u$ is replaced by $Q^{1 / 2} \frac{d}{d t} B^{H}(t)$.

We shall now verify our conditions (A1) and (A2) for this case. By the deterministic theory (see e.g. [14]) it is well known that $\left(S_{\alpha}(t), t \geq 0\right)$ is analytic and the condition (A2) (exponential stability) is satisfied in the Dirichlet case with $\delta \geq 0$ and in the Neumann case with $\delta>0$. In order to verify the assumption (A1) we have to check the behaviour of the operator $N$. It is known that $N \in \mathcal{L}\left(U, V_{\varepsilon}\right)$ (with $U=L^{(\partial \mathcal{O})}$ and $\left.V=L^{2}(\mathcal{O})\right)$ for

$$
\varepsilon<\frac{1}{4}
$$

in the case of Dirichlet boundary conditions and

$$
\varepsilon<\frac{3}{4}
$$

for the Neumann boundary conditions. Assuming that the covariance $Q$ is trace class (if $d=1$ this is automatically satisfied, because $U=\mathbf{R}^{2}$ ), the condition (A1) is satisfied by Corollary 2.2 if $1-\varepsilon<H$, which yields

$$
H>\frac{3}{4}
$$


in the Dirichlet case and

$$
H>\frac{1}{4}
$$

in the Neumann case.

To summarise, if $\delta \geq 0$ and $H>3 / 4$ (in the Dirichlet case) or $\delta>0$ and $H>1 / 4$

(in the Neumann case), then Theorems 3.1, 3.5, 3.7 and 4.1 may be applied to the problems (17),(18),(19) or (17),(18),(20).

\section{REFERENCES}

[1] E. Alòs, O. Mazet, And D. Nualart, Stochastic calculus with respect to Gaussian processes, Ann. Probab., 29:2(2001), pp. 766-801.

[2] G. Da Prato and J. Zabczyk, Ergodicity for infinite-dimensional systems, London Mathematical Society Lecture Note Series, vol. 229, Cambridge University Press, Cambridge, 1996.

[3] L. Decreusefond And A. S, Üstünel, Stochastic analysis of the fractional Brownian motion, Potential Anal., 10:2(1999), pp. 177-214.

[4] T. E. Duncan, B. Maslowski, and B. Pasik-Duncan, Fractional Brownian motion and stochastic equations in Hilbert spaces, Stoch. Dyn., 2:2(2002), pp. 225-250.

[5] _ Stochastic Equations in Hilbert Space with a Multiplicative Fractional Gaussian Noise, Stoch. Process. Appl., 115:8(2005), pp. 1357-1383.

[6] _ Linear stochastic equations in a Hilbert space with a fractional Brownian motion, International Series in Operations Research \& Management Science, vol. 94, pp. 201-222, Springer Verlag, 2006.

[7] W. Grecksch And V. V. Anh, A parabolic stochastic differential equation with fractional Brownian motion input, Statist. Probab. Lett. 41:4(1999), pp. 337-346.

[8] Y. Hu, Integral transformations and anticipative calculus for fractional Brownian motions, Mem. Amer. Math. Soc., 175:825(2005), viii+127.

[9] M. Huebner And B. L. RozovskiI, On asymptotic properties of maximum likelihood estimators for parabolic stochastic SPDE's, Prob. Theory and Relat. Fields 103(1995), pp. 143-163.

[10] R. Khasminski and G. N. Milstein, On estimation of the linearized drift for nonlinear stochastic differential equations, Stoch. Dyn., 1:1(2001), pp. 23-43.

[11] B. Maslowski and D. NuAlart, Evolution equations driven by a fractional Brownian motion, J. Funct. Anal. 202:1(2003), pp. 277-305.

[12] B. MAsLowski AND J. PospíšIL, Ergodicity and parameter estimates for infinite-dimensional fractional Ornstein-Uhlenbeck process, submitted to Appl. Math. Optim.

[13] B. Maslowski and B. Schmalfuss, Random dynamical systems and stationary solutions of differential equations driven by the fractional Brownian motion, Stochastic Anal. Appl. 22:6(2004), pp. 1577-1607.

[14] A. PAZY, Semigroups of linear operators, Springer, New York, 1983.

[15] J. PospíšLL, On parameter estimates in stochastic evolution equations driven by fractional Brownian motion, Ph.D. thesis, University of West Bohemia in Plzeň, 2005, pp. iv +88 .

[16] _ Numerical approaches to parameter estimates in stochastic differential equations driven by fractional Brownian motion, Proceedings of the Programms and algorithms of numerical mathematics 13, 2006, Prague.

[17] _ Numerical parameter estimates in stochastic equations with fractional Brownian mo- 
tion, Proceedings of the Numerical Analysis and Approximation Theory, 2006, ClujNapoca, Romania, July, 2006, pp. 353-364.

[18] B. L. S. Prakasa Rao, Estimation for some stochastic partial differential equations based on discrete observations. II, Calcutta Statist. Assoc. Bull. 54(2003), pp. 215-216, 129-141.

[19] _ Parametric estimation for linear stochastic differential equations driven by fractional Brownian motion, Random Oper. Stochastic Equations 11:3(2003), pp. 229-242.

[20] - Identification for linear stochastic systems driven by fractional Brownian motion, Stochastic Anal. Appl. 22:6(2004), pp. 1487-1509.

[21] Yu. A. Rozanov, Stationary random processes, Holden-Day, San Francisco, 1966. 